\title{
Development and Characterization of Gear Shape Porous Scaffolds Using 3D Printing Technology
}

\author{
Muhammad Hilmi Jalil1,2*, Mitsugu Todo ${ }^{3}$ \\ 1 Interdisciplinary Graduate School of Engineering Sciences, Kyushu University. 6-1 Kasuga Koen, Kasuga, \\ Fukuoka, 816-8580, Japan. \\ 2 Universiti Malaysia Pahang,Pahang, Malaysia. \\ ${ }^{3}$ Research Institute of Applied Mechanics, Kyushu University, Japan. \\ * Corresponding author. Email: hilmijalil99@gmail.com \\ Manuscript submitted November 1, 2016; accepted January 3, 2017. \\ doi: 10.17706/ijbbb.2017.7.2.74-83
}

\begin{abstract}
Continuous porous structures of biodegradable polylactic acid (PLA) were fabricated using a rapid prototyping machine with the three dimensional fused deposition modeling (FDM) technique. Effects of two different circle packing methods, the square (SQ) and the hexagonal (HEX) packings, and different pore diameters on the compressive mechanical properties were examined. The compression test results showed that SQ1 and HEX1 with $1 \mathrm{~mm}$ pore diameter had the largest compressive properties, suggesting that the microstructures were well constructed compared to the other specimens. Although SQ0.7 and HEX0.7 exhibited the lowest porosities, the modulus values were lowest, indicating that the microvoids degraded the stiffness of the structures. Scanning electron microscopy of the damaged regions suggested that microcracks were generated along the interlayers or within the layers due to bending deformation and the final fracture were initiated with these microcracking mechanism. It is thus concluded that the fabrication process must be improved so that the microcrack formation is minimized. Finite element analysis was used as an evaluation tools by comparing the experimental compressive modulus and a good agreement was exhibited correspondingly.
\end{abstract}

Key words: Compressive property, microcracking, polylactic acid, scaffold, 3D printer.

\section{Introduction}

Polylactic acid (PLA) has been considered as an excellent scaffold material in tissue engineering. Scaffolds basically have porous structures for cell proliferation, and therefore, a typical structure of scaffolds consists of pores and struts. Pores are supposed to be continuous inside of the scaffolds because cells are cultured and grown within the continuous pores. The struts support mechanical loading and hence, the mechanical properties strongly depend on the strut structure. In a mechanically optimized scaffold, the struts are constructed along the loading direction. The simplest structure of scaffold with mechanical optimization is the unidirectional porous structure where continuous pores and struts are constructed in the loading direction. Actually, this kind of unidirectional porous structure has effectively been utilized for a bone substitute, where bone growth takes place in the direction of the pores and maximized stiffness and strength are obtained in that direction [1], [2].

Several fabrication methods such as solvent casting, melt molding and rapid prototyping (RP) have been applied to produce such porous structures [3]. One kind of RP method is the fused deposition modeling 
(FDM) technique, which has been managed to provide enhanced control over scaffold shape and pores properties such as porosity and pore size [4]. Furthermore, PLA can be used as the source material by introducing 3D printing (3DP) using FDM technique. 3DP is also known to have excellent ability to control matrix architecture, design and material composition. A variety of materials are used to fabricate porous scaffold structures using 3DP machines. For example, polymeric molds were fabricated by 3DP to make porous alumina and tricalcium phosphate ceramic structures [5]. Recent development of feedstock material and hardware enhancement enabled PLA to be used as the source material of 3DP, directly to produce porous structures. It is worth noting that some 3DP machines, e.g. used in the present study, guarantee the fabrication of PLA porous structures without using additional chemical modification and support material during fabrication process and without using any following processes to remove solvent or powder from the final specimens. However, little attention has been paid to correlate the relationship between the porous design and the mechanical properties of porous scaffolds fabricated by 3DP. Furthermore, Finite Element Method (FEM) was chosen as the evaluation tool to assist in the prediction of mechanical characterization of the scaffolds. Capability of FEM in predicting mechanical behavior of multilayer systems was showed in a different fabrication method using robocast scaffolds by [6].

In this study, unidirectional porous PLA specimens with three different pore diameters were fabricated using a commercial 3DP machine with FDM technique. Two different packing methods of pores were also employed and furthermore, gear shape was adapted as the cross-sectional design because the side grooves are thought to be effective for enhancing the adhesion between the scaffold surface and the surrounding tissue when it is implanted into human body. Effects of pore size and packing method on the compressive mechanical properties were then assessed. Fracture micromechanisms of the specimens were also characterized by observing the damage regions using a field-emission scanning electron microscope. Along with experimental results, FEM analysis was also performed in order to predict basic mechanical properties such as the compressive modulus and strength of the scaffolds.

\section{Material and Method}

The main material used in this study was a biodegradable thermoplastic polymer, polylactic acid (PLA) filament (Kenbill Co.) with diameter $1.75 \mathrm{~mm}$ and natural colored. The melting temperature is around $190-220^{\circ} \mathrm{C}$ with density $1.288 \mathrm{~g} / \mathrm{cm}^{3}$. A commercial 3D printer developed based on the FDM technique (Scoovo C170-S, Open Cube Inc.) was used to fabricate porous cylindrical PLA structures using the PLA filament. This machine can print polymer with the minimum thickness of $100 \mu \mathrm{m}$. It has 1 print head nozzle of diameter $0.4 \mathrm{~mm}$, and can print any models within a dimension of $175 \mathrm{~mm}$ height $\mathrm{x} 150 \mathrm{~mm}$ width $\mathrm{x} 150$ $\mathrm{mm}$ length. The machine is also equipped with some useful functions, such as extrusion width, advanced infill setting and sequential printing, which can be adjusted to obtain specific print results.

The unidirectional porous structures were designed using a computer aided design (CAD) software. Two different kinds of circle packing method, i.e. hexagonal and square, were used to design the distribution of pores. The hexagonal method (HEX) is known to be the densest packing of circles in the 2 dimension plane [7]. This packing method, also known as bee's honeycomb, is shown in Fig. 1(a). The latter square method (SQ) has a sparse packing and can be a good example for comparison. The different in the circle density eventually affects the porosity and mechanical characteristics of each specimen. A cylindrical solid specimen without pores was also fabricated as the control specimen.

The specimen's diameter and height were both $8 \mathrm{~mm}$. The pores were cylindrical with diameter, $d_{\text {pore }}$ of 2 $\mathrm{mm}, 1 \mathrm{~mm}$ and $0.7 \mathrm{~mm}$ for both the packing methods. Therefore, 6 different specimens were fabricated and thereafter denoted as SQ2, SQ1, SQ0.7, HEX2, HEX1 and HEX0.7, respectively. Porosity and pore distribution of the scaffolds are also affected by the distance between adjacent circular pores, $l$. In this study, by taking 
the nozzle diameter $(0.4 \mathrm{~mm})$ into account, the distance $l$ was determined as $1.5 \mathrm{~mm}$ for all the specimens. The designs were exported as rapid prototyping stereolithography format (.stl file format) and uploaded into the 3DP software (Slic3r ${ }^{\mathrm{TM}}$ v. 0.9.10b). The models were then sliced into layers and a G-code file, which defined the movement of the printer's extruder and other setting, was created and sent to the 3D printer machine. The integrated dispensing system of the printer automated the deposition of PLA material through XYZ axial space. Other parameters in the system such as the printing temperature, bed temperature and layer height were also controlled.

Finally, 2D sliced layers were built on X-axis orientation, where continuous strut was deposited layer by layer by the printer. The processor of the 3DP translated the dimensions of the structures into $\mathrm{X}, \mathrm{Y}$ and $\mathrm{Z}$ coordinates, and controlled the nozzle following the calculated path. During fabrication, the first layer played an important role to determine the binding of the next layer with the printing bed. By default, the first layer height should be around $150 \%$ from the normal layer height. A thicker first layer was important to improve adhesion and tolerance for non-perfect build plates. The printing temperature was fixed at $220^{\circ} \mathrm{C}$, and the nozzle travel speed was set at $60 \mathrm{~mm} / \mathrm{s}$. The overviews of the fabricated porous specimens are shown in Figs. 2 and 3.

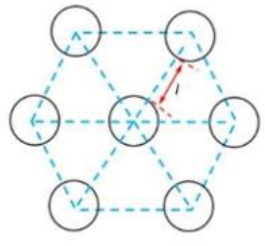

(a)

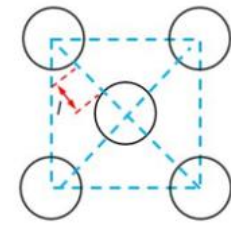

(b)

Fig. 1. (a) Hexagonal and (b) square packing method. where, $l$ is the distance between two circles in closest proximity.

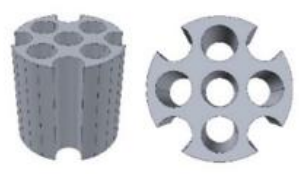

(a)

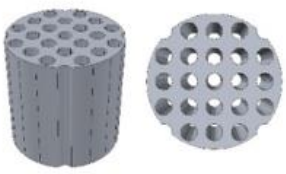

(b)

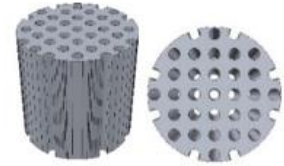

(c)

Fig. 2. Isometric and axial view of CAD models with cylindrical pore packed in square pattern.

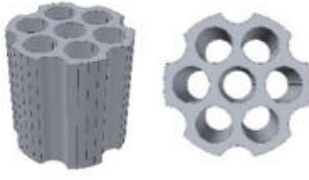

(a)

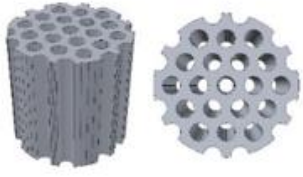

(b)

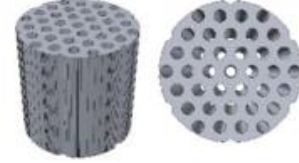

(c)

Fig. 3. Isometric and axial view of 3D CAD models with cylindrical pore packed in hexagonal pattern.

\section{Analysis and Testing}

\subsection{Porosity}

The theoretical porosity of volume percentage, $V_{\text {porosity,th, was }}$ calculated for each specimen using the initial deposition geometries based on a unit cube, assuming that the strut diameter and spacing between layers were equal (i.e., no overlap caused by the merging between struts from one layer to another). 


$$
\begin{gathered}
V_{\text {porosity,th }}=\left(1-\frac{V_{t}}{V_{a}}\right) \times 100 \% \\
V_{t}=\frac{\pi d^{2} L_{f i l}}{4} \\
V_{a}=\frac{\pi D^{2} H}{4}=1608.5 \mathrm{~mm}^{3}
\end{gathered}
$$

where $V_{t}$ is the true volume $\left(\mathrm{mm}^{3}\right), V_{a}$ is the apparent volume $\left(\mathrm{mm}^{3}\right) . d, L_{f i l}, D$ and $H$ refer to the filament diameter, length of filament consumed during printing process, diameter and height of porous cylinder, respectively [8]. The experimental porosity, $V_{\text {porosity, exp }}$, was also evaluated by

$$
\begin{gathered}
\text { Vol }_{\text {porosity,exp }}=\left(1-\frac{\rho_{\text {exp }}}{\rho_{\text {solid }}}\right) \times 100 \% \\
\rho_{\text {exp }}=\frac{w_{\text {exp }}}{V_{a}}\left(g \cdot \mathrm{mm}^{-3}\right)
\end{gathered}
$$

where $\rho_{\text {solid }}, \rho_{\text {exp }}$ and $w_{\text {exp }}$ are the density of solid PLA $\left(=1.288 \times 10^{3} \mathrm{~g} \cdot \mathrm{mm}^{-3}\right)$, the density and the weight of porous specimen, respectively.

\subsection{Compression Test}

Compression tests were carried out using a conventional testing machine (Shimadzu EZ-L) equipped with a $5 \mathrm{kN}$ load cell at a constant crosshead speed of $1 \mathrm{~mm} \mathrm{~min}^{-1}$. Tests were performed in the direction perpendicular to the printing plane of the scaffolds. Force and stroke were measured and recorded in a personal computer. Average engineering stress and strain values were then calculated from the force and stroke. The equivalent compressive modulus and the compressive strength were evaluated as the slope of the initial linear region and the maximum value of stress in the stress-strain curve, respectively.

\subsection{Microscopic Observation}

Printed and the fractured specimens were placed on aluminum plates and coated with Pt-Pd using an Ion Sputter machine (Hitachi E-1030). Then the coated surfaces were observed using a field-emission scanning electron microscope (FE-SEM) (Hitachi S-4100). Morphological observation of the axial section of the printed structures was also conducted using a digital microscope (Vitiny UM-06).

\section{FEM Analysis}

FEM simulations were carried out using, Mechanical Finder ${ }^{\mathrm{TM}}$ 7.0 Extended edition software (Research Center of Computational Mechanics, Inc Japan). The algorithm modeled a cylindrical porous structure of the same dimension as printed specimens, diameter $8 \mathrm{~mm}$ and height $8 \mathrm{~mm}$. Two different architectures were designed as shown in Fig. 2 and 3: a) a hexagonal packing pores with three various $d_{\text {pore }}, 2 \mathrm{~mm}, 1 \mathrm{~mm}$ and $0.7 \mathrm{~mm}$ and b) square packing pores with the same $d_{\text {pore }}$ various. The analysis was done in aiming to understand the fracture behavior under uniaxial compression stress $(5000 \mathrm{~N})$ and to evaluate the experimental data by comparing each compressive modulus. As shown in Table 1, the properties from the control scaffolds was used as the material properties in this analysis.

A compressive loading condition was simulated with a uniform load of $5000 \mathrm{~N}$ along vertical direction was applied on the top of the models. The boundary conditions of the models was determined as fixation of the bottom of each porous cylindrical model in all displacements directions. 
Table 1. Material Properties for FEA

\begin{tabular}{ccccc}
\hline \hline Properties & $\begin{array}{c}\text { Young`s modulus } \\
(\mathrm{MPa})\end{array}$ & $\begin{array}{c}\text { Compressive strength } \\
(\mathrm{MPa})\end{array}$ & $\begin{array}{c}\text { Density } \\
\left(\mathrm{g} / \mathrm{mm}^{3}\right)\end{array}$ & $\begin{array}{c}\text { Poisson } \\
\text { ratio }\end{array}$ \\
\hline Experimental & 1458.96 & 231.2 & $2.9 \times 10^{-4}$ & 0.4 \\
\hline \hline
\end{tabular}

\section{Result and Discussion}

\subsection{Porosity and Dimension}

Table 2 showed the comparison between theoretical and experimental porosity of each type of specimens obtained using (1) and (4). Overall, all samples displayed decent value of porosity which may allow cell adhesion, proliferation and distribution as a scaffold geometry. Although high porosity in excess $90 \%$ was chosen in many scaffold fabrication and design since it allows better tissue culturing [9], an intermediary value which balance with the mechanical strength of scaffolds was assessed in this study. The highest theoretical porosity was shown by specimen SQ2 with 86.9\%, while in experimental, HEX2 showed highest porosity of $90.7 \%$. In contrast to [4] that argued about the possibility of fusion between struts was the reason for lower porosity in experimental. However, this study showed such possibility did not occur. A possible reason could be during the fabrication process, deposited struts cooled down enough before the next adjacent layer of struts was dispensed, making sure that no fusion occur between adjacent struts.

Table 2. Theoretical and Experimental Porosity of Unidirectional Porous Specimens

\begin{tabular}{l|c|c|c|c}
\hline \multirow{3}{*}{ Type } & $\begin{array}{c}\text { Pore diameter } \\
(\mathbf{m m})\end{array}$ & $\begin{array}{c}\text { Theoretical Porosity } \\
\mathbf{( \% )}\end{array}$ & $\begin{array}{c}\text { Experimental Porosity } \\
\mathbf{( \% )}\end{array}$ & $\begin{array}{c}\text { Experimental } \boldsymbol{d}_{\text {pore }} \\
\text { (mm) }\end{array}$ \\
\hline \multirow{3}{*}{$\begin{array}{l}\text { Square } \\
(S Q)\end{array}$} & 2 & 86.9 & 89.5 & $2.025 \pm 0.175$ \\
\cline { 2 - 5 } & 1 & 82.3 & 86.5 & $0.865 \pm 0.065$ \\
\hline \multirow{2}{*}{$\begin{array}{l}\text { Hexa } \\
\text { (HEX) }\end{array}$} & 0.7 & 77.5 & 85 & $0.62 \pm 0.12$ \\
\cline { 2 - 5 } & 2 & 82.3 & 90.7 & $1.85 \pm 0.15$ \\
\hline
\end{tabular}
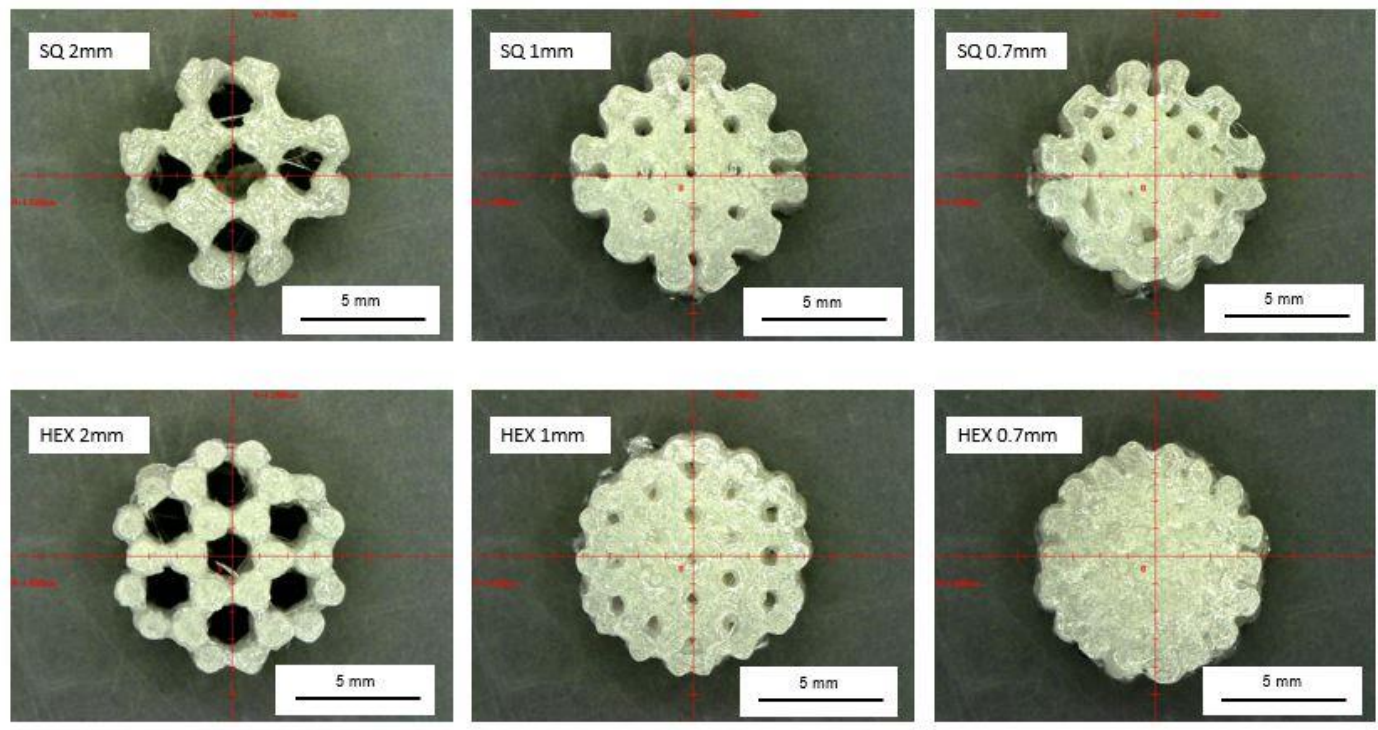

Fig. 4. Axial view of the printed specimens of SQ and HEX.

Preliminary test was done to optimize the dimensional measurement of printed specimens. Looking into 
the diameter and height of the porous structures, it was noted that the size of printed parts accurately matched the CAD design. By observing the digital microscope images of axial view of all specimens, the printed porous scaffold displayed sufficient porous for $2 \mathrm{~mm}$ and $1 \mathrm{~mm}$ specimens, while $0.7 \mathrm{~mm}$ specimens showed poor porous presence. According to Fig. 4 and Table 2, the diameter of unidirectional porous, $d_{\text {pore }}$ for SQ2, SQ1, SQ0.7, HEX2, HEX1 and HEX0.7 were approximately 1.85 to $2.2 \mathrm{~mm}, 0.8$ to $0.93 \mathrm{~mm}, 0.5$ to $0.74 \mathrm{~mm}, 1.7$ to $2 \mathrm{~mm}, 0.8$ to $0.93 \mathrm{~mm}$, and 0.25 to $0.4 \mathrm{~mm}$, respectively.

Poor fabrication of porous in SQ0.7 and HEX0.7 may due to the printing parameter and deposition nozzle's limitation. A review [10] mentioned that practically only scaffolds with pores larger than $0.5 \mathrm{~mm}$ have been fabricated to date, despite the advancement of 3DP technology's potential. Fine tuning on the printing speed, layer height and temperature were suggested for improving printing result. Overall, it was clear that larger pore diameter while maintaining uniform pore distance resulted in higher porosity of the specimen. Additionally, at present, structures with pore diameter less than $0.7 \mathrm{~mm}$ was difficult to produce due to machine limitations.

\subsection{Compression Properties}

The compressive modulus and strength are shown in Fig. 5. For comparison, the modulus and strength of the solid bulk specimen are also shown in the figure. It was apparent that SQ1 and HEX1 showed the highest modulus and strength in each group. Obviously SQ1 and HEX1 had lower porosities than SQ2 and HEX2, resulting in the higher properties. On the contrary, although SQ0.7 and HEX0.7 possessed lower porosities than SQ1 and HEX2, they exhibited lower properties. This might be due to the poor fabrication of these specimens, where the deposition speed of the strut was too high to create pores with the smallest diameter of $0.7 \mathrm{~mm}$; as a result, the binding between layers became poor. It is therefore suggested that adjustment of the deposition speed with other printing parameters needs to be optimized for each design, although it will affects overall time consumption.

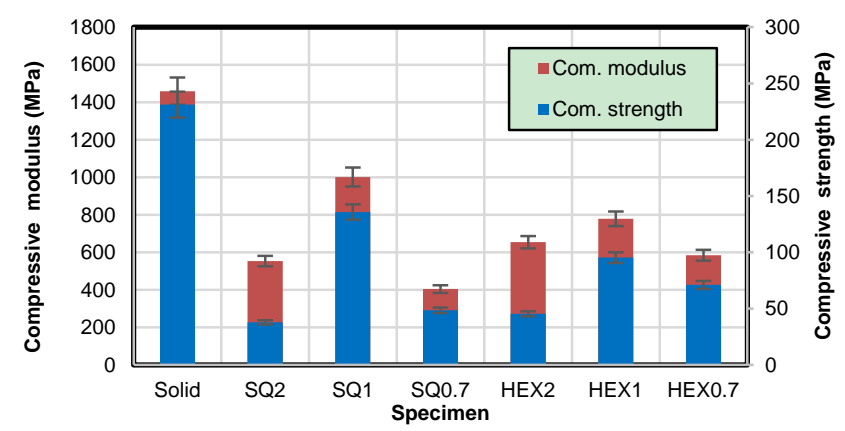

Fig. 5. Compressive modulus and strength.

As expected, the compressive properties of HEX2 and HEX0.7 were higher than those of SQ2 and SQ0.7, respectively since the porosity values of HEX specimens were lower than those of SQ specimens. However, SQ1 exhibited higher properties than those of HEX1, although the porosity of SQ1 was lower. This suggests that the inner structure of SQ1 was firmly constructed more than HEX1 that might have more gaps between the layers. Such effect of porosity on the mechanical properties was also explained in different structures of bidirectional pores of the orthogonal and the displaced layer [4]. Many previous studies discussed that the optimization of the printing parameter involved complex interactions between the hardware, software and material properties [11]. Each aspect of 3DP has to be tuned and customized in order to successfully obtain the best 3D architecture for each specific application [12].

\subsection{Microstructures and Failure Modes}


SEM images of the pores on the top surface are shown in Fig. 6. It is seen from Figs. 6 (b) and (e) that the unidirectional holes were clearly fabricated in SQ1 and HEX1 using the 3DP. On the other hand, in SQ0.7 and HEX0.7, the diameters of pores were scattered and in SQ2 and HEX2, rough surfaces were observed along the edges of the pores. Except SQ2, $d_{\text {pore }}$ values of the printing specimens were tended to be smaller than the designed sizes of the CAD models. In the present study, the 3DP system was able to fabricate precise size of porous structure when the pore size was larger than $1 \mathrm{~mm}$.

SEM images of the side-surfaces of the specimens damaged by the compression tests are shown in Fig. 7. It is seen that microcracks were generated between the strut layers in the vertical direction or in the interlayers for both specimens. It is thought that these cracks were generated due to bending deformation of the layers or shear deformation between the layers and therefore, assuming an opening fracture mode (Mode 1) will generate cracking perpendicular to the loading direction. SEM images of these regions at a higher magnification are shown in Fig. 8. As shown in these figures, smaller microcracks were also generated on the surfaces of the layers. The larger microcracks shown in Fig. 7 were thought to be formed as the coalescence of these microcracks.

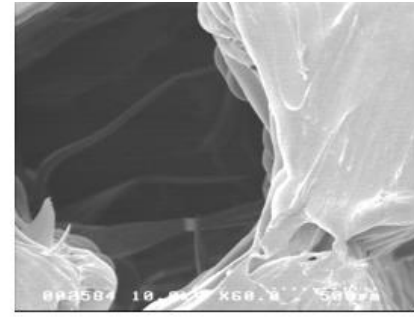

(a) SQ2

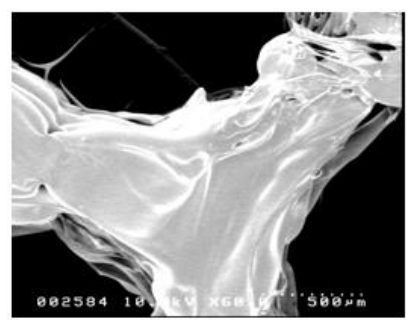

(d) HEX2

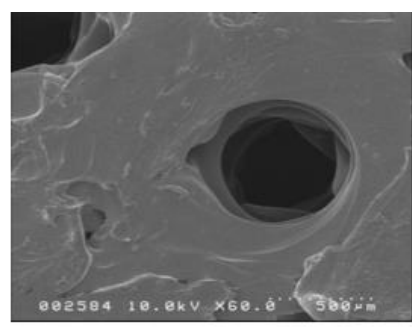

(b) SQ1

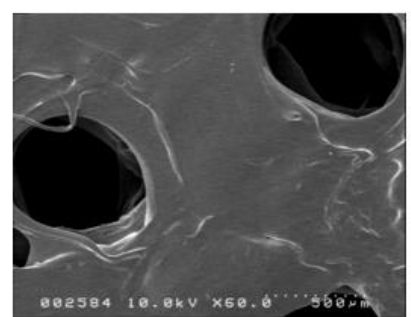

(e) HEX1

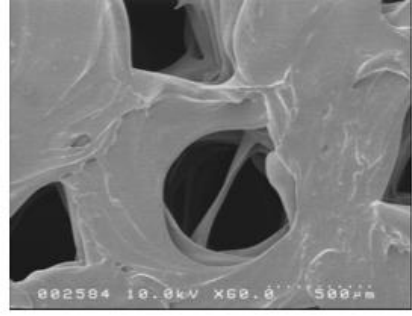

(c) SQ0.7

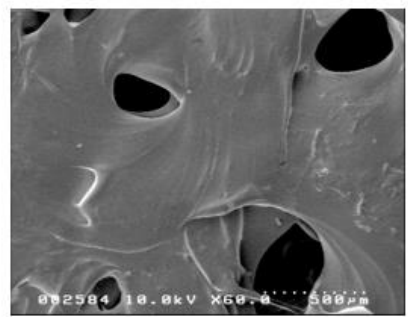

(f) HEX0.7

Fig. 6. SEM images of the top surfaces of SQ and HEX specimens.

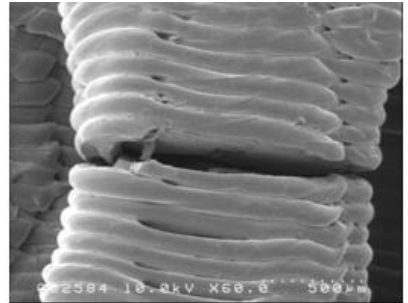

(a) SQ2

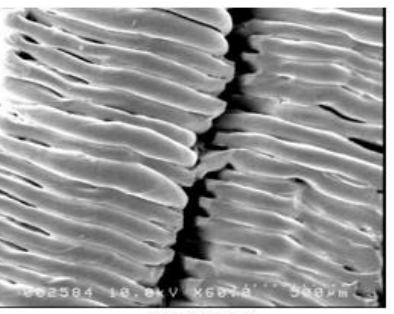

(d) HEX2

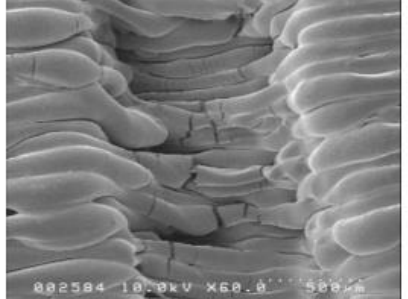

(b) SQ1

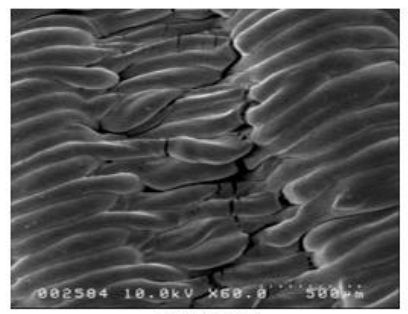

(e) HEX1

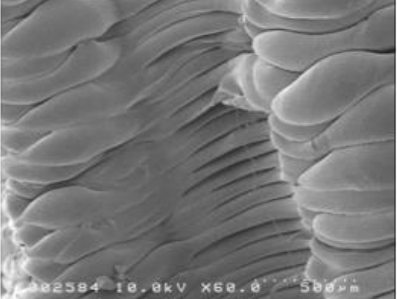

(c) SQ0.7

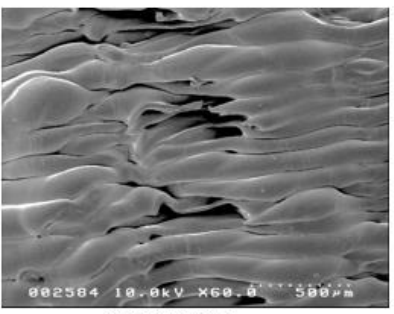

(f) HEX0.7

Fig. 7. SEM images of layered regions of SQ and HEX specimens after compression testing. 


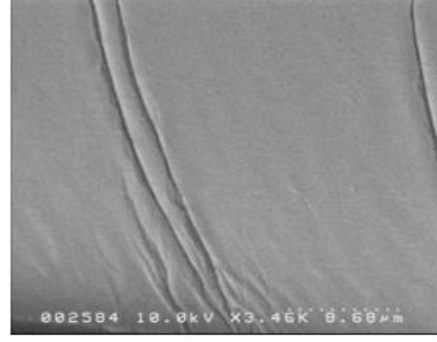

(a) SQ2

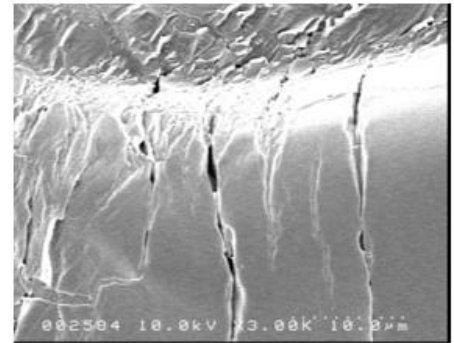

(d) HEX2

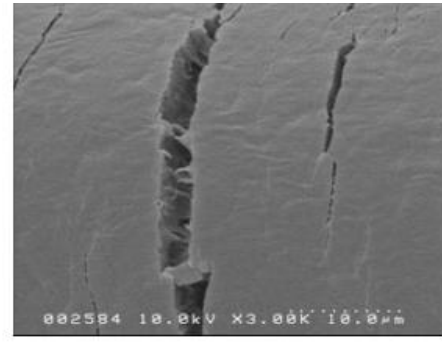

(b) SQ1

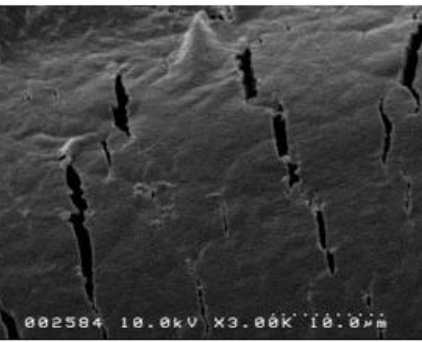

(e) HEX1

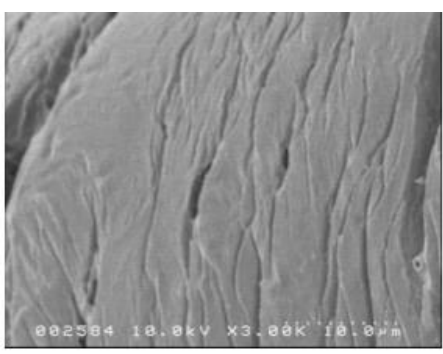

(c) SQ0.7

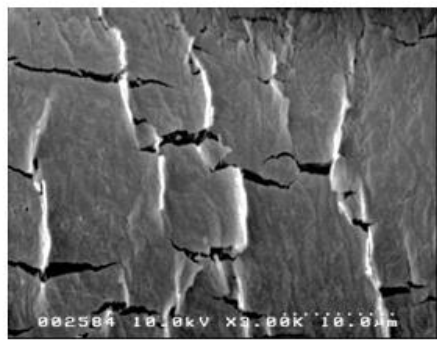

(f) HEX0.7

Fig. 8. FE-SEM images of micro-cracking regions of SQ and HEX specimens.

\subsection{FEA Result}

In this section, the validity of FEM was discussed by comparing between the compressive modulus and strength predicted by the FEM and experimental results. As shown in Fig. 9, modulus value of FEM for all type of scaffold were slightly smaller than experimental excluding for HEX1. The relative difference between experimental and FEM modulus range in between $58.9 \%$ to $-7.8 \%$. Overall, the modulus value obtained from the FEM were significantly in good agreement with the experimental. HEX2 showed the largest relative difference with the FEM value 58.9\% smaller than the experimental.
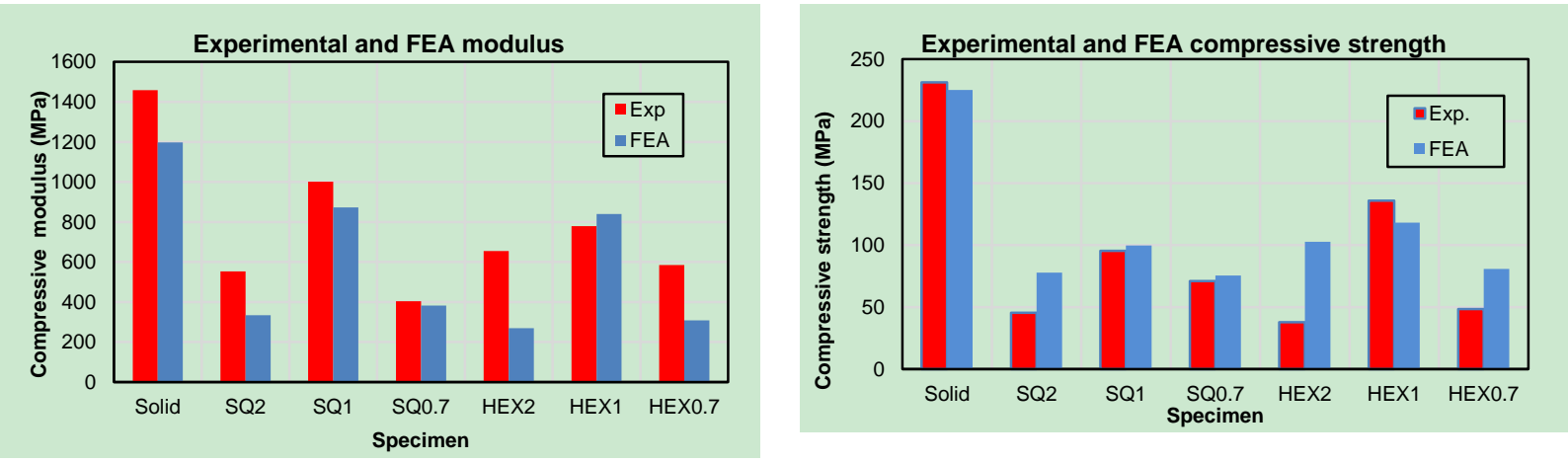

Fig. 9. Comparison between experimental and FEA modulus and compressive strength.

It is simple to understand that FEM prediction of compressive strength is slightly higher than the experimental result excluding for scaffold Solid and HEX0.7. The relative difference of compressive strength between experimental and predicted FEM range in between $172 \%$ to $-12 \%$. HEX2 significantly showed overestimation value of FEA of $172 \%$ larger than experimental. Apparently, unlike the compressive modulus result, FEM overestimated the compressive strength of the specimens. These could be attributed to the rapid crack growth in the actual experiment, where it could be increasing the strength and modulus of the scaffold. This can be seen in the case of slower crosshead speed, $0.6 \mathrm{~mm} \mathrm{~min}^{-1}$, used during uniaxial compression by [6] which resulted in slow crack growth and decreased strength. Another reason could be induced by the modulus applied in the FEM which was obtained from uniaxial compression test of the 
controlled bulk specimen. Although the geometry and size of the bulk specimen was analogous with the real situation, the fabrication nature of the scaffolds which come in a rod-shaped strut might suggest a different approach to obtain the material property, i.e., three-point bending test. In sum, these result confirm that FEM could be applied as an excellent evaluation tools to predict the structural strength of simple scaffolds.

\section{Conclusion}

In this study, a good agreement between FEA and experimental results were achieved by observing 2 critical structural characteristics which were the compressive modulus and strength. Certainly, predictions of compressive modulus and others mechanical criterion using the FEA could be a great game-changing tool in optimizing the mechanical performance of scaffold fabricated using 3DP through design and geometry.

\section{Acknowledgment}

This work was supported by JSPS KAKENHI Grant Number JP15K13836.

\section{References}

[1] Iwasashi, M., Funayama, T., Watanabe, A., Noguchi, H., Tsukanishi, T., Suetsugu, Y., Makihara, T., Ochiai, N., Yamazaki, M., \& Sakane, M. (2015) Bone regeneration and remodeling within a unidirectional porous hydroxyapatite bone substitute at a cortical bone defect site: histological analysis at one and two years after implantation. Materials, 8(8), 4884-4894.

[2] Noguchi, H., Watanabe, A., Funayama, T., Tsukanishi, T., Wadano, Y., \& Sakane, M. (2013). A novel unidirectional porous hydroxyapatite cylinder implanted in the dorsal muscles of dogs promotes fibrous tissue vascularization and invasion. Key Engineering Materials, 530, 275-278.

[3] Hutmacher, D. W. (2000). Scaffolds in tissue engineering bone and cartilage. Biomaterials, 21(24), 2529-2543.

[4] Serra, T., Planell, J. A., \& Navarro, M. (2013).High-resolution PLA-based composite scaffolds via 3-D printing technology. Acta biomaterialia, 9(3), 5521-30.

[5] Bose, S., Darsell, J., Kintner, M., Hosick, H., \& Bandyopadhyay, A. (2003).Pore size and pore volume effects on alumina and TCP ceramic scaffolds. Materials Science and Engineering C, 23(4), 479-486.

[6] Miranda, P., Pajares, A., \& Guiberteau, F. (2008). Finite element modeling as a tool for predicting the fracture behavior of robocast scaffolds. Acta biomaterialia, 4(6), 1715-24.

[7] Graham, R. L., Lubachevsky, B. D., Nurmela, K. J., \&. Östergård, P. R. J. (1998). Dense packings of congruent circles in a circle. Discrete Mathematics, 181, 139-154.

[8] Woodfield, T. B. F., Malda, J., De Wijn, J., Péters, F.. Riesle, J., \&. Van Blitterswijk, C. A. (2004). Design of porous scaffolds for cartilage tissue engineering using a three-dimensional fiber-deposition technique. Biomaterials, 25(18), 4149-4161.

[9] Polo-Corrales, L., Latorre-Esteves, M., \& Ramirez-Vick, J. E. (2014). Scaffold design for bone regeneration. Journal of nanoscience and nanotechnology, 14(1), 15-56.

[10] Butscher, A., Bohner, M., Hofmann, S., Gauckler, L., \& Müller, R. (2011). Structural and material approaches to bone tissue engineering in powder-based three-dimensional printing. Acta Biomaterialia, 7(3), 907-920,

[11] Zein, I., Hutmacher, D. W., Tan, K. C., \& Teoh, S. H. (2002). Fused deposition modeling of novel scaffold architectures for tissue engineering applications. Biomaterials, 23(4), 1169-1185.

[12] Asadi-Eydivand, M., Solati-Hashjin, M., Farzad, A., \& Abu Osman, N. A. (2016). Effect of technical parameters on porous structure and strength of 3D printed calcium sulfate prototypes. Robotics and Computer-Integrated Manufacturing, 37, 57-67. 


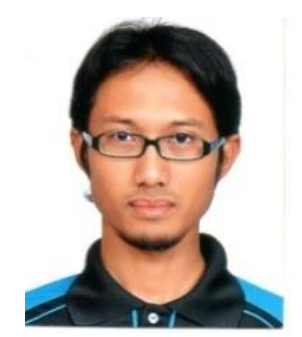

Muhammad Hilmi Jalil was born in Johor, Malaysia. He obtained his bachelor degree of mechanical engineering and the master of applied medical engineering sciences from the Yamaguchi University, Japan. Currently, he is pursuing his $\mathrm{PhD}$ at the Kyushu University, Japan in the biomaterial field. He is receiving a fellowship from Faculty of Mechanical Engineering, Universiti Malaysia Pahang, Malaysia.

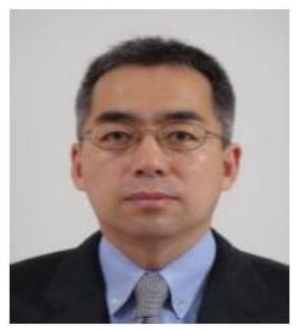

Mitsugu Todo obtained his bachelor and master's degrees of engineering from Kyushu University, Japan, and PhD from the Ohio State University, the United States of America. $\mathrm{He}$ is currently an associate professor of Research Institute for Applied Mechanics, Kyushu University, and working on biomaterials for osteochondral tissue engineering and biomechanics of orthopedic implant. 